\title{
Quality of life, symptoms and pulmonary function in asthma: long-term treatment with nedocromil sodium examined in a controlled multicentre trial
}

\author{
P.W. Jones and the Nedocromil Sodium Quality of Life Study Group
}

Quality of life, symptoms and pulmonary function in asthma: long-term treatment with nedocromil sodium examined in a controlled multicentre trial. P.W. Jones and the Nedocromil Sodium Quality of Life Study Group. CERS Journals Ltd 1994.

ABSTRACT: This study was designed to measure improvement in quality of life of patients with asthma, using a standardized disease-specific questionnaire, the $\mathrm{St}$ George's Respiratory Questionnaire, in a year long double-blind, placebo-controlled, group comparative study with nedocromil sodium.

Two other questionnaires were used: the Sickness Impact Profile (a measure of general health) and the Hospital Anxiety and Depression Scale. Measurements were made at baseline and following 24 and 48 weeks of treatment. Response to therapy was also evaluated using daily diary card and peak flow measurements, clinic assessments and spirometry. Following a 4 week baseline, 719 adult asthmatics were randomized to treatment with $4 \mathrm{mg}$ nedocromil sodium or placebo. Patients currently maintained on inhaled corticosteroids received treatments four times daily, those on bronchodilator alone received treatments twice daily.

The Impacts component of the St George's Respiratory Questionnaire was significantly improved in patients receiving nedocromil sodium, as were night-time asthma, asthma severity at clinic, and daytime inhaled bronchodilator use. In patients receiving placebo, most of the traditional variables improved, and all three questionnaires recorded significant improvements in health. Patients and clinicians judged nedocromil sodium more effective than placebo. The improvement in St George's Questionnaire score in the nedocromil sodium treated patients was approximately double the change considered to be clinically significant.

The study has shown that improvements in health with prophylactic therapy for asthma may be quantified by the use of a standardized disease-specific questionnaire. Eur Respir J., 1994, 7, 55-62.
*Nedocromil Sodium Quality of Life Study Group (participants listed below).

Correspondence: P.W. Jones

Division of Physiological Medicine

St George's Hospital Medical School

Cranmer Terrace

London SW17 0RE

UK

Keywords: Asthma nedocromil sodium quality of life

randomized controlled clinical trial

Received: September 231992

Accepted after revision September 211993
Treatment efficacy in asthma has traditionally been determined by measurement of symptoms and pulmonary function; however, there is evidence for a complex relationship between these and the patient's overall health [1]. Over the last $30 \mathrm{yrs}$, there has been much research into the development and validation of questionnaires designed to quantify the impact of disease on daily life and well-being from the patient's point of view. The first of these were comprehensive instruments to measure the effect of a wide range of different disease states [2, 3]. They appear to provide reliable estimates of health in patients with airflow limitation [3, 4], but may be relatively insensitive for patients with mild to moderate disease [1]. There has also been some concern that these general measures may be relatively insensitive to changes in disease state $[5,6]$.

In recent years, a number of disease-specific measures have been developed. In order to produce an instrument that was sensitive to changes in health, some of these have allowed a degree of "individualization". This approach may produce a sensitive questionnaire, but one that lacks the advantages conferred by standardization, particularly the ability to compare directly results from different studies or study populations. One disease-specific measure, the St George's Respiratory Questionnaire (SGRQ), was designed to be standardized and sufficiently sensitive to detect and measure the size of any change in health following treatment $[7,8]$. The SGRQ was included in

*Nedocromil Sodium Quality of Life Study Group: R.J.C. de Amaral-Marques (Lisbon, Portugal); M.F. Bone (Dudley, UK); L-P. Boulet (Quebec, Canada); V. Brusasco (Genova, Italy); C.M. Burke (Dublin, Ireland); A.J. Carvalheira-Santos (Lisbon, Portugal); G. Ciappi (Rome, Italy); R.J. Cordier (Brussels, Belgium); E. Crimi (Genova, Italy); N. Del Bono (Pisa, Italy); M. De Vos (Ghent, Belgium); T.K-A. Ekström (Linkoping, Sweden); C. Franco (Massa Marittima, Italy); R. Fuangtong (Bangkok, Thailand); S.N. Furman (Pinelands, South Africa); P. Godard (Montpellier, France); M. Havu (Kotka, Finland); P. Howard (Sheffield, UK); P.W. Jones (London, UK); K. Kiviranta (Helsinki, Finland); S. Koskinen (Kiljava, Finland); P.A. Kuusisto (Tampere, Finland); J.H. Levenstein (Illinois, USA); W.T. McNicholas (Dublin, Ireland); F-B. Michel (Montpellier, France); M.J. Paananen (Jyväskylä, Finland); A. Stewart (Sheffield, UK); U.G. Svendsen (Copenhagen, Denmark); R.L.M. Tammivaara (Kiljava, Finland); S. Valente (Rome, Italy); K.K.K. Venho (Savonlinna, Finland); J. Westbroek (Heerenveen, Holland); P. Youngchaiyud (Bangkok, Thailand). 
this, the first placebo-controlled study of the effect of prophylactic therapy on daily life and well-being in patients with chronic asthma. The study lasted one year, and used the SGRQ, together with the Sickness Impact Profile [2], and the Hospital Anxiety and Depression Scale [9]. Traditional measures of treatment effectiveness (diary card variables and clinic visit data) were also recorded. The treatment used was nedocromil sodium, a preventive agent, the efficacy and safety of which in asthma has been established from long-term [10] and placebo-controlled [11-13] studies.

This study provided a test of the ability of a standardized measure of health, such as the SGRQ, to quantify improvements in health following therapy for asthma.

\section{Methods}

The study was a 48 week, double-blind, group comparison of nedocromil sodium (Tilade $\AA$ ) and placebo. Twenty six centres recruited 719 patients into a 4 week baseline. The centres were drawn from 14 countries: Belgium (45 patients), Canada (13), Denmark (28), Finland (142), France (6), Holland (9), Ireland (31), Italy (181), Portugal (18), South Africa (90), Sweden (21), Thailand (48), UK (53), and USA (34).

The patients were assigned to either Group A (inhaled corticosteroid plus other therapy, $n=456$ ) or Group B (inhaled and/or oral bronchodilators, $n=263$ ), and randomly allocated to receive two inhalations of $2 \mathrm{mg}$ nedocromil sodium or matching placebo via metered dose inhaler. Group A patients received treatments four times daily and Group B received treatments twice daily. Randomization was designed to ensure a balance of placebo and nedocromil sodium treated patients in each group within each participating centre. Patients receiving hyposensitization, sodium cromoglycate or $\geq 10 \mathrm{mg} \cdot \mathrm{day}^{-1}$ prednisolone (or equivalent) were not allowed into the study. Patients completed daily diary cards to record daytime and nighttime asthma symptoms (scale range: $0=$ none, $4=$ very severe), the highest of three measurements of morning and evening peak expiratory flow (PEF) and medication use (test treatment, day and night-time inhaled bronchodilator use and other medication). Clinic visits were as follows: admission, end of baseline, at 4 week intervals for 12 weeks, and at 6 week intervals thereafter. At the end of baseline and subsequent visits the clinician recorded asthma severity since the previous visit $(0=$ none, $1=$ mild, $2=$ moderate, $3=$ severe and $4=$ =very severe), pulmonary function (forced expiratory volume in one second $\left(\mathrm{FEV}_{1}\right)$, forced vital capacity (FVC) and PEF) and acute exacerbations or unusual symptoms. Patients were asked not to use an inhaled bronchodilator for at least $4 \mathrm{~h}$ before a visit. At the final visit, the clinician and patient recorded their opinion of test treatment $(1=$ very effective to $5=$ made condition worse).

Quality of life was assessed from three questionnaires: the St George's Hospital Respiratory Questionnaire (SGRQ) [8], the Sickness Impact Profile (SIP) [14], and the Hospital Anxiety and Depression Scale (HAD) [9]. These were completed during the baseline, and after 24 and 48 weeks of treatment. The SGRQ comprises 76 weighted responses $[15,16]$ to a range of questions, divided into: Symptoms (distress caused by specific respiratory symptoms); Activity (physical activities that cause or are limited by breathless-ness); and Impacts (social and psychological effects of the disease). A Total score is derived from all items. The SIP contains 136 items grouped into 12 categories of health-related problems. The scores from all categories are aggregated and expressed as a percentage of the maximum possible, to produce a total score. The scoring range for the SIP and SGRQ is $0-100$. The HAD contains 14 questions (seven separately assessing anxiety and depression), answered using a 0-3 scale. With all three questionnaires, a high score indicated poor health, so that a decrease in score indicated an improvement in quality of life. Patients also completed a five point scale for overall health (GH5), the categories of which were: very poor; poor; fair; good; and very good. This was used to test for differences in interpretation or completion of the questionnaires between countries. Its simplicity minimized ambiguities arising in translation. All questionnaires originated in English. Those administered in non-English speaking countries were translated, then backtranslated into English by a second translator, to check that the sense of each question had been adequately transferred. Each questionnaire was checked for adequacy of completion. The study purpose was explained to all patients, who gave their written or verbal consent. The protocol was approved at all centres by the appropriate Local Ethics Committee.

\section{Statistical analysis}

All assessments were by direct comparison with placebo, using change from baseline. Baseline clinic data were those collected at the end of baseline visit. Mean values for diary card data were calculated from the baseline,

Table 1. - Patient characteristics

\begin{tabular}{|c|c|c|}
\hline & Nedocromil sodium & Placebo \\
\hline $\operatorname{Sex} \quad M / F$ & $178 / 190$ & $150 / 198$ \\
\hline NR & 0 & 3 \\
\hline Age yrs* & $\begin{array}{c}44(12-77) \\
n=367\end{array}$ & $\begin{array}{c}44(12-73) \\
n=348\end{array}$ \\
\hline Asthma duration yrs* & $\begin{array}{c}14(1-65) \\
n=367\end{array}$ & $\begin{array}{c}15(1-64) \\
\mathrm{n}=348\end{array}$ \\
\hline Severity over last year & & \\
\hline Mild & 22 & 25 \\
\hline Moderate & 194 & 193 \\
\hline Severe & 122 & 106 \\
\hline Very severe & 21 & 16 \\
\hline NR & 9 & 11 \\
\hline $\mathrm{FEV}_{1} \%$ pred $^{* *}$ & $\begin{array}{l}68 \pm 27 \\
n=362\end{array}$ & $\begin{array}{l}68 \pm 28 \\
n=342\end{array}$ \\
\hline $\mathrm{FEV}_{1} \% \mathrm{rev}^{* *}$ & $\begin{array}{l}26 \pm 18 \\
n=363\end{array}$ & $\begin{array}{l}27 \pm 21 \\
n=346\end{array}$ \\
\hline
\end{tabular}

*: data presented as mean, and range in parenthesis; **: data presented as mean $\pm S D$. Sample size is given when values are missing. $\mathrm{FEV}_{1}$ : forced expiratory volume in one second; NR: not recorded; M: male; F: female; \% pred: percentage predicted; $\%$ rev: percentage reversibility to a standard dose of inhaled bronchodilator e.g. $200 \mu \mathrm{g}$ salbutamol. 
periods between clinic visits, and the treatment period as a whole (weeks 1-48). Data from the SGRQ were analysed in patients aged $\geq 20$ yrs (nedocromil sodium, $\mathrm{n}=338$; placebo, $\mathrm{n}=331$ ) since the questionnaire had not been validated for use in patients below that age. Parametric tests were used throughout [17]. For each variable, twoway analyses of variance were used with treatment, Group and treatment $\times$ Group interaction as factors to test for differences in response to nedocromil sodium between the two patient types included in the study. Supplementary analyses were carried out within Groups A and B using Student's t-tests. Analysis of variance was also performed on all baseline quality of life scores, to test the association between these scores and the GH5 measure. Country and the GH5 × country interaction were added as factors to this analysis, to test for differences in the relationship between quality of life score and GH5 between countries. All tests were two-tailed and a p-value of $<0.05$ was considered significant.

\section{Results}

\section{Patients and compliance}

Patient entry characteristics were similar between treatment groups (table 1). A good match between treatments was also maintained when the patients were classified into Groups A and B (mean age 47 and 47 yrs in Group A; 39 and 38 yrs in Group B; mean duration of asthma 14.5 and 16.0 yrs in Group A; 13.2 and 13.7 yrs in Group $\mathrm{B}$; for nedocromil sodium and placebo treated patients, respectively). There were no significant treatment $x$ Group interactions for any variable for any period of analysis, except night-time asthma during weeks 19-24, and FVC at the week 18 and week 24 clinic visits. The data from Groups A and B were, therefore, combined in an overall analysis.

Twenty percent of patients (145) failed to complete the study. These withdrawals were evenly distributed (18-25\%) across the patient groupings, irrespective of test treatment, time on test treatment, or existing therapy. Test treatment recorded using diary cards over the four and six week periods between clinic visits suggested a high level of compliance. Mean use (inhalations per day $\pm \mathrm{SD}$ ) for weeks $1-48$ was $7.5 \pm 1.1$ and $7.4 \pm 1.2$ (Group

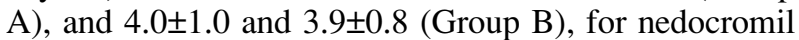
sodium and placebo, respectively.

\section{Quality of life}

Baseline scores for the SGRQ (see legend to fig. 1) showed a moderately high impact of asthma on the patients' lives. The SIP score was mildly elevated (table 2 ), and the Anxiety and Depression scores were relatively low (table 2), well below the clinically significant level [9]. Patients treated with either nedocromil sodium or placebo recorded decreases in quality of life scores, irrespective of existing therapy. Scores from the SGRQ a)

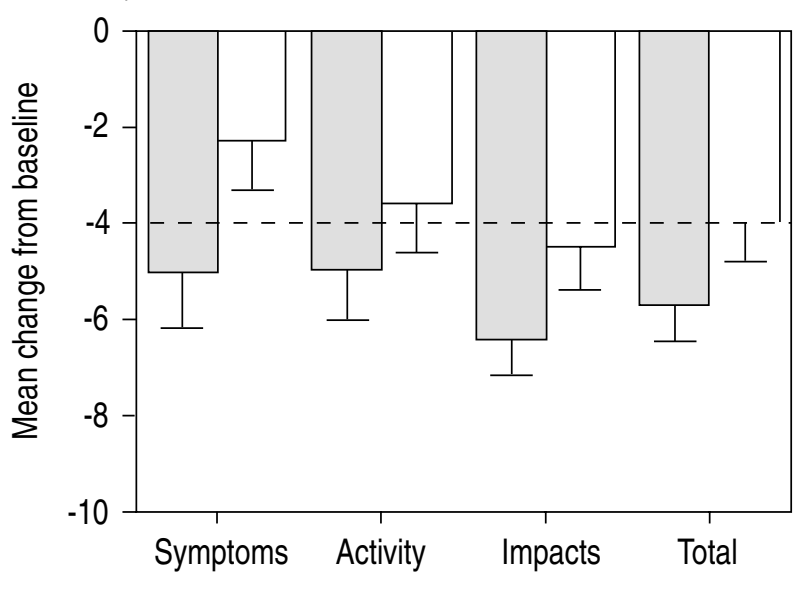

b)

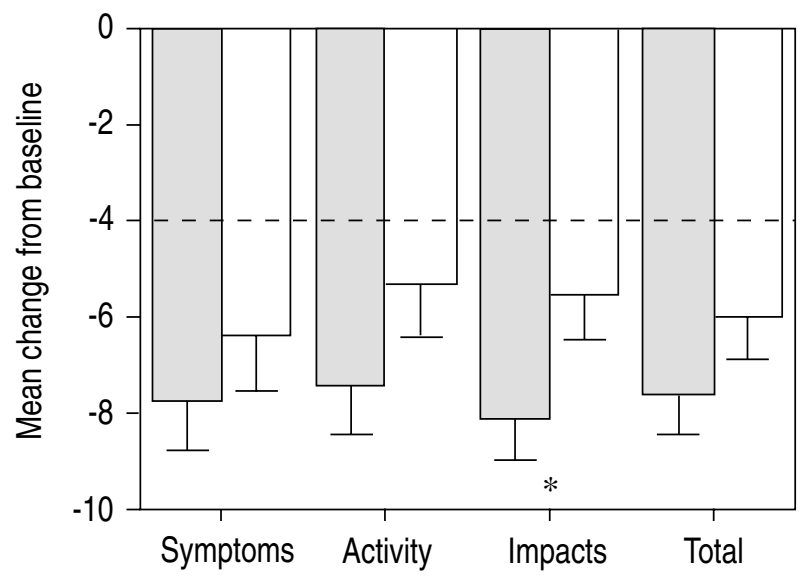

Fig. 1. - Mean change from baseline in the St George's Hospital Respiratory Questionnaire scores: a) after 24 weeks of treatment; b) after 48 weeks of treatment. Error bars in the figure are SEMs. $\square$ : nedocromil sodium; $\square$ : placebo. Baseline scores, as mean \pm SEM (nedocromil sodium/placebo): Symptoms 55.2 $\pm 0.9 / 54.3 \pm 0.9$; Activity $39.0 \pm 1.1 / 38.1 \pm 1.1$; Impacts $34.1 \pm 1.0 / 31.8 \pm 0.9$; Total $39.3 \pm 0.9 / 38.0 \pm$ 0.8 . The dashed line indicates the threshold level of significance [7]. *: $\mathrm{p}<0.05$ nedocromil sodium $v s$ placebo.

Table 2. - Sickness Impact Profile (SIP) and Hospital Anxiety and Depression (HAD) scores at baseline, and at 24 and 48 weeks of treatment

\begin{tabular}{lrr}
\hline & Nedocromil sodium & Placebo \\
\hline HAD - Anxiety & & \\
Baseline & $6.7 \pm 3.9$ & $6.8 \pm 4.0$ \\
Week 24-Ba & $-0.8 \pm 2.9$ & $-0.7 \pm 2.9$ \\
Week 48-Ba & $-1.0 \pm 3.0$ & $-0.7 \pm 3.3$ \\
HAD - Depression & & \\
Baseline & $4.6 \pm 3.3$ & $4.4 \pm 3.3$ \\
Week 24-Ba & $-0.5 \pm 2.9$ & $-0.2 \pm 2.7$ \\
Week 48-Ba & $-0.6 \pm 2.7$ & $-0.4 \pm 2.7$ \\
SIP - Total & & $6.3 \pm 6.2$ \\
Baseline & $6.3 \pm 6.7$ & $-0.8 \pm 4.9$ \\
Week 24-Ba & $-1.1 \pm 4.6$ & $-1.3 \pm 5.4$ \\
Week 48-Ba & $-1.2 \pm 4.5$ & \\
\hline
\end{tabular}

Scores are presented as mean $\pm \mathrm{SD}$. Ba: baseline. 
(fig. 1), HAD and SIP (table 2) generally decreased to a greater extent in nedocromil sodium treated patients. After 24 weeks, decreases in scores for all the quality of life measures in the nedocromil sodium treated patients were highly significant $(\mathrm{p}<0.001)$. In the placebo group over the same period, there were falls in all components of the SGRQ: Symptoms $(\mathrm{p}<0.05)$, Impacts, Activity, Total (all $\mathrm{p}<0.01)$. The Anxiety and SIP scores also fell significantly (both $\mathrm{p}<0.01$ ). The fall in Depression was not significant after 24 weeks, but it was at 48 weeks. By the end of the study, SGRQ scores had improved (i.e. decreased) by approximately eight points with nedocromil sodium treatment and 5-6 points in placebo treated patients. The SGRQ Impacts score was significantly $(\mathrm{p}<0.05)$ more improved in nedocromil sodium treated patients compared with the placebo group (fig. 1). As a general observation, the decrease in quality of life scores in placebo treated patients at the end of the study was approximately equivalent to the decreases in nedocromil sodium treated patients at the interim assessment.

\section{Nationality and quality of life assessment}

All of the quality of life scores differed between countries $(\mathrm{p}<0.01)$. To test whether the questionnaires were completed in different ways in different countries, the GH5 was used as a reference measure of health, which, because of its simplicity and lack of ambiguity, should have been used by patients very similarly in all participating countries. There was a significant linear trend between baseline quality of life scores and the GH5 score $(\mathrm{p}<0.0001)$. When country was introduced as a factor into this analysis, the interaction between country and GH5 score was significant only with the Anxiety and Depression scores from the HAD $(\mathrm{p} \leq 0.02)$, but not with the SGRQ or SIP scores $(\mathrm{p}>0.05)$. This suggests that cultural or linguistic factors may have influenced the patients' responses to the mood state questionnaire, but not the other quality of life measures.

\section{Diary card variables}

Mean asthma symptom severity during the baseline period was mild to moderate for all patients (see legend to fig. 2). Asthma symptom scores improved both in placebo and nedocromil sodium treated patients, but more so with nedocromil sodium treatment.

Night-time asthma severity was significantly reduced in the nedocromil sodium treated patients for the majority of the treatment phase (fig. 2). Comparison with placebo over the entire 48 week period showed a significant $(\mathrm{p}<0.01)$ reduction in night-time asthma severity with nedocromil sodium (table 3 ). a) Daytime asthma

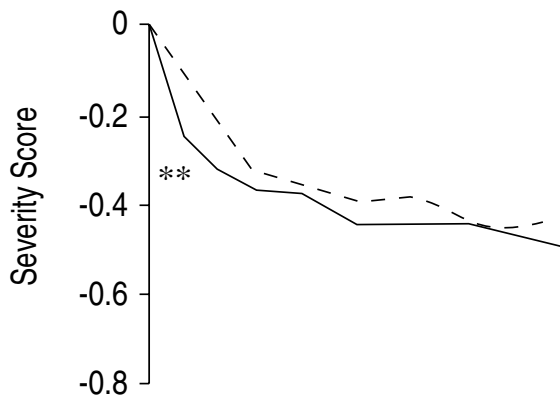

b) Night-time asthma

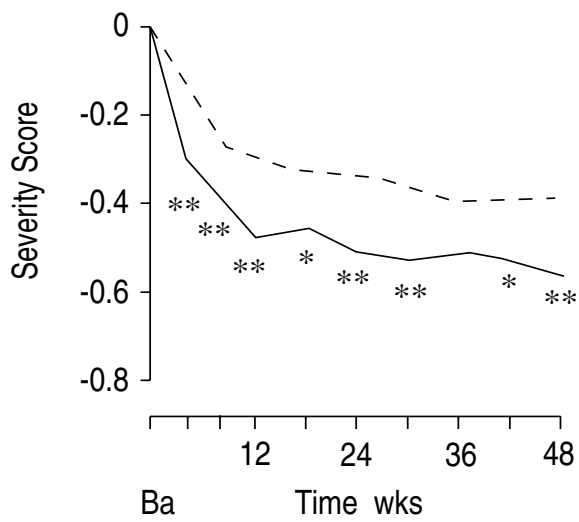

c) Morning PEF

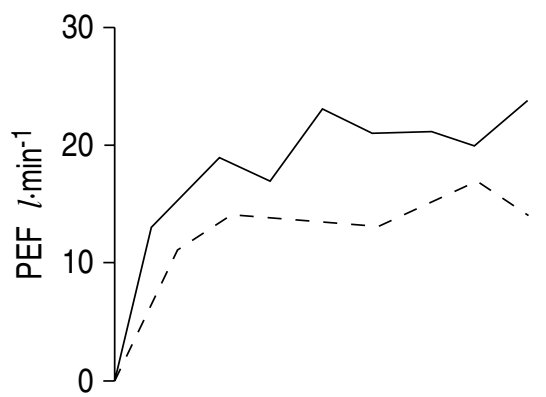

d) Evening PEF

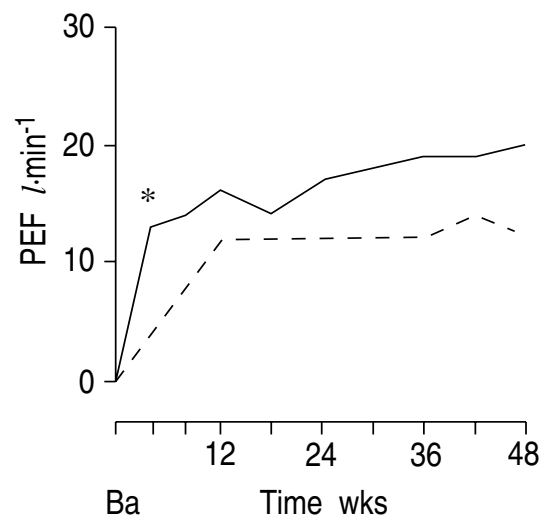

e) Daytime $\beta_{2}$ use

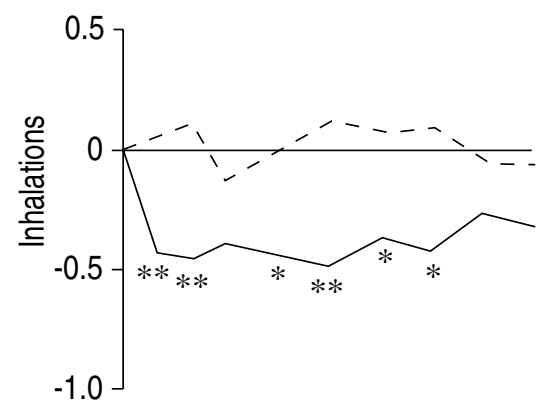

f) Night-time $\beta_{2}$ use

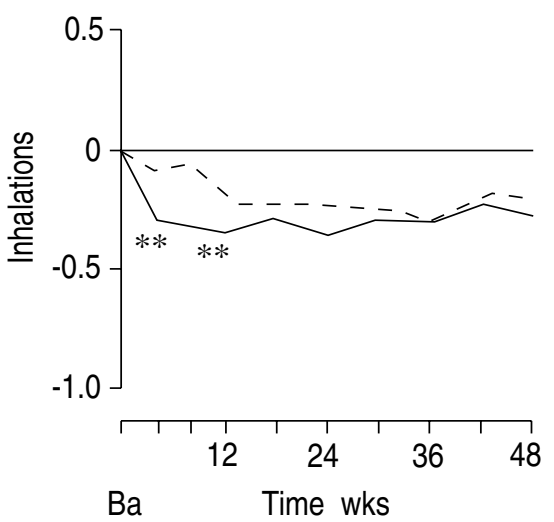

Fig. 2. - Mean change from baseline (Ba) for diary card variables: a) Daytime asthma; b) Night-time asthma; c) Morning PEF; d) Evening PEF; e) Daytime bronchodilator use; f) Night-time bronchodilator use. _ _ : nedocromil sodium; - - - : placebo. Baseline values (nedocromil

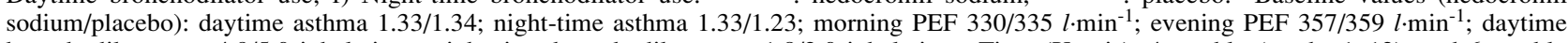
bronchodilator use 4.9/5.0 inhalations; night-time bronchodilator use 1.8/2.0 inhalations. Time (X-axis): 4 weekly (weeks $1-12$ ); and 6 weekly (weeks 13-48) periods between clinic visits. *: $\mathrm{p}<0.05 ; * *$ : $<<0.01$ nedocromil sodium $v s$ placebo at time point shown. PEF: peak expiratory flow. 
Table 3. - Diary card asthma score and bronchodilator use: mean change over 48 weeks of treatment compared with baseline

\begin{tabular}{|c|c|c|c|}
\hline & $\begin{array}{l}\text { Nedocromil } \\
\text { sodium }\end{array}$ & Placebo & $\begin{array}{c}\text { Treatment effect } \\
\text { p value }\end{array}$ \\
\hline \multicolumn{4}{|c|}{ Daytime asthma score } \\
\hline Baseline & $1.33 \pm 0.64$ & $1.34 \pm 0.71$ & \\
\hline Change & $-0.39 \pm 0.55$ & $-0.32 \pm 0.61$ & 0.14 \\
\hline \multicolumn{4}{|c|}{ Night-time asthma score } \\
\hline Baseline & $1.33 \pm 0.77$ & $1.23 \pm 0.75$ & \\
\hline Change & $-0.47 \pm 0.65$ & $-0.33 \pm 0.63$ & $<0.01$ \\
\hline \multicolumn{4}{|c|}{ Daytime bronchodilator use (inhalations) } \\
\hline Baseline & $4.9 \pm 3.4$ & $5.0 \pm 3.4$ & \\
\hline Change & $-0.4 \pm 2.0$ & $0.0 \pm 2.1$ & 0.01 \\
\hline \multicolumn{4}{|c|}{ Night-time bronchodilator use (inhalations) } \\
\hline Baseline & $1.8 \pm 1.6$ & $2.0 \pm 3.1$ & \\
\hline Change & $-0.3 \pm 1.1$ & $-0.2 \pm 1.5$ & 0.31 \\
\hline
\end{tabular}

Data are presented as mean \pm SD.

Mean baseline morning and evening PEF (see legend to fig. 2) was consistent with the mild to moderate severity of symptoms. Improvements from baseline were observed with both test treatments, but were always greater with nedocromil sodium (fig. 2). PEF tended to improve fairly rapidly over the first 12 weeks, then continued to increase steadily in the nedocromil sodium group but levelled off with placebo treatment.

Patients treated with nedocromil sodium had an immediate, significant $(\mathrm{p}<0.01)$ decrease in daytime and night-time bronchodilator use (fig. 2). Daytime use remained significantly reduced throughout the majority of the study in nedocromil sodium treated patients, but did not alter from baseline in placebo treated patients. Daytime bronchodilator use was significantly $(\mathrm{p}=0.01)$ reduced with nedocromil sodium compared with placebo treatment over the 48 week treatment period (table 3 ). The night-time difference in bronchodilator use was not sustained beyond the first 12 weeks.

Mean daily inhaled corticosteroid use in Group A was similar between nedocromil sodium and placebo treated patients during the baseline and all periods of analysis $(\mathrm{p} \geq 0.3$ ). Oral bronchodilator use in Group B (mean doses per day \pm SD during the baseline: nedocromil sodium 1.5 \pm 2.6 ; placebo $1.3 \pm 2.1$ ) decreased throughout in patients treated with nedocromil sodium (mean reduction $21 \%$ over weeks 1-48) compared with little or no change in the placebo group $(2 \%)$.

\section{Clinic assessments}

Asthma assessed in the clinic was judged to be moderately severe at baseline (see legend to fig. 3). Greater improvements were observed with nedocromil sodium compared with placebo treatment throughout the study (fig. 3). Mean improvements from baseline over the whole treatment period (nedocromil sodium 0.59; placebo, 0.44) were significantly $(\mathrm{p}<0.01)$ in favour of nedocromil sodium. Mean changes in spirometry at clinic visits were generally greater with nedocromil sodium treatment but tended to be small $(\leq 5 \%)$ and with few significant differences (fig. 3). a)

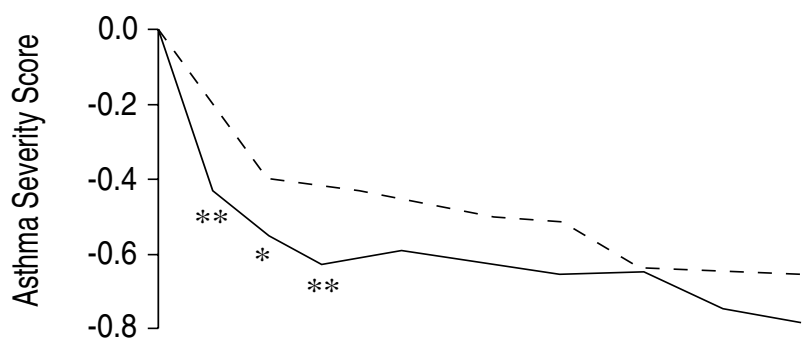

b)

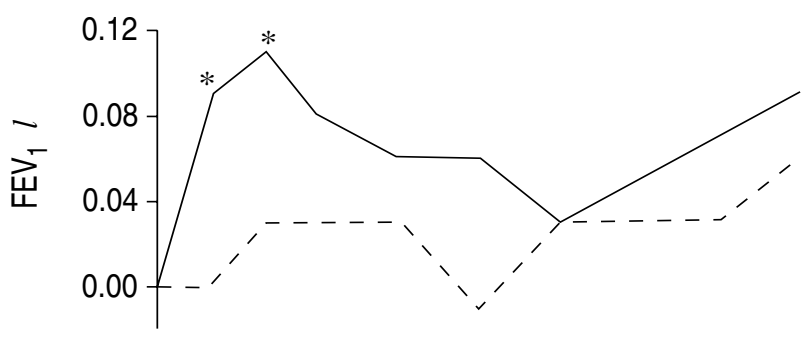

c)

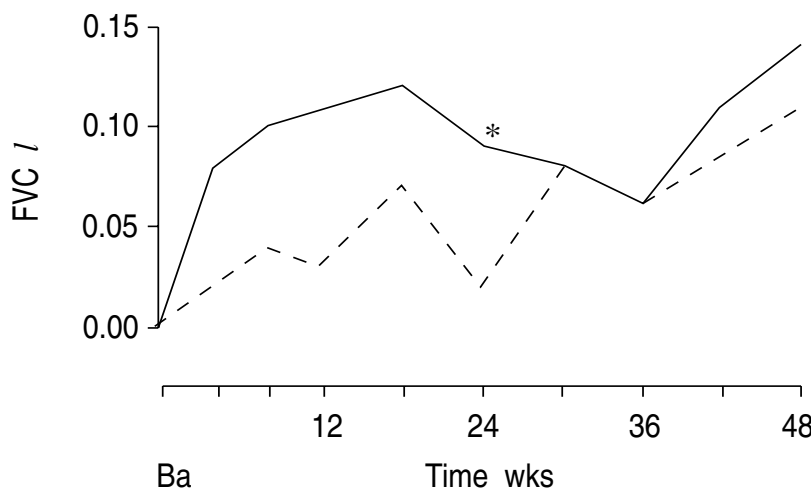

Fig. 3. - Mean change from baseline (Ba) for variables recorded in the clinic. a) Asthma severity; b) forced expiratory volume in one second $\left(\mathrm{FEV}_{1}\right)$; c) forced vital capacity (FVC). — : nedocromil sodium; - - - : placebo. Baseline values (nedocromil sodium/ placebo): asthma severity score 2.0/2.0; FEV $12.1 / 2.2$; FVC 3.1/3.1. Time (X-axis): 4 weekly (weeks $1-12$ ); and 6 weekly (weeks 13-48) periods between clinic visits. $*$ : $\mathrm{p}<0.05 ; * *: \mathrm{p}<0.01$ nedocromil sodium $v s$ placebo. 
Sixty five percent $(n=194)$ of nedocromil sodium treated patients considered their treatment to be very or moderately effective, whilst $54 \%$ of placebo treated patients $(n=153)$ reached the same conclusion. The clinicians considered that $58 \%(\mathrm{n}=177)$ of nedocromil sodium treated patients and $44 \%$ of placebo treated patients $(n=123)$ had received a very or moderately effective treatment. Mean patient and clinician opinion scores were significantly $(\mathrm{p} \leq 0.0005)$ in favour of nedocromil sodium.

\section{Exacerbations}

A large number of exacerbations were recorded by nedocromil sodium treated patients (965) and those receiving placebo (967). The principal cause was recorded as infection (nedocromil sodium 41\%; placebo 42\%). These figures also include exacerbations during the baseline (i.e. prerandomization phase) of the study. The exacerbation rate in the winter months was over $40 \%$ higher than in the remainder of the year. The physicians prescribed additional medication to $65 \%$ of patients receiving nedocromil sodium (62\% with placebo).

\section{Tolerability}

Nedocromil sodium and placebo were well-tolerated. Thirteen patients (five taking nedocromil sodium) withdrew owing to chest-related symptoms (e.g. cough, bronchospasm, wheeze) and four (three on nedocromil sodium) owing to nausea. Unusual taste was reported more frequently $(9.5 \%)$ in nedocromil sodium treated patients compared to placebo treated $(2.3 \%)$, but only two nedocromil sodium treated patients withdrew because of taste.

\section{Discussion}

We have reported the first placebo-controlled study of prophylaxis in asthma in which quality of life instruments have been used. In view of the novelty of the study, three different questionnaires were selected. The SIP was used because it is an established measure of general health and was included in two National Institutes of Health studies in airways disease, the Intermittent Positive Pressure Breathing study [18] and the Nocturnal Oxygen Therapy Trial (NOTT) [19]. The HAD was incorporated to specifically address mood state disturbances. This questionnaire contains relatively few items on the physical consequences of anxiety and depression, which should have reduced any potential confusion between apparent improvements in mood following therapy and improved physical performance due to better lung function. The third questionnaire was the SGRQ [8]. This was designed to quantify the impact of airways disease on life and well-being. It is standardized throughout, so that all patients respond to exactly the same questionnaire items. This allows direct comparisons between health scores obtained in different studies and with different drugs or therapeutic modalities. In comparison, another recently developed questionnaire, the Asthma Quality of Life Questionnaire [20], is not entirely standardized in the same way. The value of standardization becomes apparent when comparing questionnaire scores from different trials. The SIP - a standardized questionnaire - has been widely used in airways disease. When the results from different published studies are combined, an inverse relationship can be seen between $\mathrm{FEV}_{1}$ and SIP score [1]. This accumulation of SIP scores allows the scores from the current trial to be set into a broader context. Normative values for the HAD are also available, a score of eight being the threshold of clinical significance for Anxiety and Depression [9]. This is the first published therapeutic trial using the SGRQ, but scores from a one year observational study in more severe patients have been published [8]. In that study, mean $\pm \mathrm{SD} \mathrm{FEV}_{1}$ was lower than in this trial at $48 \pm 23 \%$ predicted, and the total SGRQ score was proportionately higher (i.e. poorer health) at $47 \pm 20$ units.

There was a clear improvement in all measures of health which increased over time. This was seen both in placebo and nedocromil sodium treated patients. In nearly every instance, the improvement in the patients receiving nedocromil sodium was greater than that in the placebo treated group (fig. 1 and table 2). This difference achieved significance only with the SGRQ Impact score. This particular component of the SGRQ draws together effects of the disease on social function and emotional well-being, and has been shown to correlate predominantly with severity of disability, exercise tolerance, level of anxiety and frequency of wheeze [8]. The improvement in SGRQ score in the nedocromil sodium treated group exceeded the threshold for clinical significance by 24 weeks, and by 48 weeks was approximately double this level (fig. 1). The placebo treated patients reached the same threshold at 24 weeks, and exceeded it by 48 weeks.

The baseline SIP scores were low, and at a level to be expected for patients with this degree of asthma [1]. The improvement in SIP scores is therefore quite surprising. In the NOTT study, the changes in SIP score were only a little larger, despite starting from a much higher baseline [19]. Unlike nearly all the other measures in this trial, whether questionnaire or traditional, the total SIP score showed no evidence of a trend for greater improvement in the nedocromil sodium treated group. This observation is similar to findings from two recent studies in chronic obstructive airways disease, in which therapy produced significant improvements in exercise tolerance, but no change in SIP score [21, 22]. High anxiety has been reported in severe asthma [23], but baseline scores in our patients were relatively low, and fell further over the first 24 weeks of the study. Depression scores were considerably lower than for Anxiety and the corresponding falls were modest, yet they were significant in both placebo and nedocromil sodium treated groups after 48 weeks.

In the placebo treated patients, there were clinically significant improvements in the SGRQ scores and detectable falls in scores for SIP, Anxiety and Depression. These changes do not appear to be true placebo effects, because they were accompanied by improvements in a range of other measures including PEF (an improvement of 10-15 $l \cdot \mathrm{min}^{-1}$ ), diary card and clinic scores for asthma severity and night-time bronchodilator use (figs 2 and 3 and table 3 ). The size of these improvements in the placebo group 
was considerably greater than in previous studies of similar design [24]. We suggest that this resulted from the number and frequency of clinic assessments, together with good accessibility of the physicians to their patients. These factors may have produced a level of care which exceeded that normally received. This may have promoted an improved level of compliance with all treatments. In addition, a large number of exacerbations were recorded both in nedocromil sodium and placebo treated patients, principally due to infections in the winter months. Over $60 \%$ of the patients had additional medication at some point in the study, which again may reflect a higher than usual level of medical supervision.

Despite the large improvements in standard measures of therapeutic efficacy in the placebo treated patients, nedocromil sodium achieved levels of improvement similar to [12], and in many areas better than previously reported $[25,26]$. A positive response occurred within the first period of assessment, and improvement continued over the whole treatment period. Most notable was the improvement in night-time symptoms, concurrent with a reduction in daytime bronchodilator use. Mean PEF improved by 20-25l $\mathrm{min}^{-1}$ in nedocromil sodium treated patients. The clinicians' assessment of asthma severity showed a steady improvement throughout the study in both placebo and nedocromil sodium treated patients, but on average was greater in the latter. It is interesting to note that whilst differences between nedocromil sodium and placebo treated patients were not found with all the traditional measures of outcome, both patient and clinician opinions of treatment efficacy showed a highly significant difference in favour of nedocromil sodium. These opinions are a global measure and have been shown to correlate well with subjective measurements of asthma severity [27].

This was a multinational study, like many in asthma, so that it was necessary to translate the questionnaires into a number of different languages. This involves complexities of idiom, but also requires sensitivity to variations between cultures in the way in which health states are conceived or described. Even at the level of respiratory symptoms there may be problems. For example, in German there is no single word equivalent to the English word "wheeze". Careful translation may minimize the effect of such differences, and the absence of a direct equivalent for "wheeze" did not appear to influence the response rate in Germany to a questionnaire designed to study the epidemiology of asthma within Europe [28]. In a different study concerned with validation of the weights used in calculating the SGRQ scores, there were no significant differences between the weights ascribed to the different questionnaire items in countries as varied as Finland, Italy, Thailand, UK and USA. Only Holland stood out as having a higher level of distress for a given questionnaire item [16]. To minimize errors due to translation, the technique of back-translation was used for all quality of life instruments in this study. Following completion of the trial, we tested for the effect of differences in language or culture on the quality of life scores, by comparing them with the score from the GH5 scale of overall health. Previous studies in England had shown a good correlation between the GH5 and SGRQ [8]. In the current study, highly significant correlations were observed between the GH5 and scores for the HAD, SGRQ and SIP. In the case of the SGRQ and SIP, the relationship to the GH5 was stable across countries, but similar stability was not found in the case of the Anxiety and Depression scores. The detection of apparent differences in use of the HAD in different countries using this methodology gives us some confidence in concluding that linguistic influences on the completion of the SGRQ and SIP were likely to have been small. It appears that culture and language may have a greater influence on the interpretation of questionnaire items concerned with emotional feelings.

The pattern of changes in the quality of life scores between placebo and nedocromil sodium treated patients broadly followed the changes in the more traditional measures of efficacy, so that it is reasonable to ask what additional contribution did these rather complex measurements make to this study. In answer to this, it is important to recall that in a chronic disease such as asthma, there are four basic objectives of therapy: reduced mortality; modification of the natural history of the disease; fewer acute episodes; and a reduction in the impact of the disease on daily life. Quality of life instruments are designed to quantify the latter. It is a basic scientific principle that the variable of interest should be measured directly, if possible, rather than be inferred from a surrogate variable. The impact of asthma on a patient's life and well-being cannot be predicted reliably from measurements of airways obstruction and respiratory symptoms, because the relationships between them are too complex [1]. The quality of life questionnaires used in this study have been the subject of careful development and validation and can claim to measure the health states that they were designed to assess.

This still raises issues concerned with the meaning or clinical inference to be attached to their scores. With any new measurement, whether objective or subjective, a full understanding of the results can only develop with experience, principally by associating the scores obtained with the new instruments to more familiar and established measures. This paper provides one step in this process, by presenting quality of life data alongside traditional measures so that the reader may begin to make an association between the relative size of the changes in each. A more formal approach, relating change in SGRQ score to changes in other measures of disease activity has been carried out in a different group of patients [7]. This concluded that a fall in SGRQ score of four points may be judged to be worthwhile on clinical grounds. Thus, we see that in the current study, the threshold of clinically significant improvements in health was exceeded by patients receiving placebo, and with nedocromil sodium the improvements were approximately double this threshold value. It is more difficult to identify thresholds for clinically significant responses with traditional measures of therapeutic efficacy. Furthermore, there may be problems in formulating an estimate of the overall benefit to the patient when each variable may change to a different degree and some may not change at all. 
In conclusion, this study has shown that the SGRQ, a standardized disease-specific measure, could quantify improvements in health following prophylactic therapy for asthma with nedocromil sodium. The SIP, a comprehensive general measure, detected the improvement in health seen in all patients in the trial, but failed to identify the difference between nedocromil sodium and placebo treated patients. The SGRQ appeared to be used similarly in different countries despite differences in language and culture, although the mood state questionnaire scores did appear to be influenced by nationality. Quality of life measurements complement data from traditional measures of outcome, and may provide valuable summative estimates of overall treatment efficacy.

Acknowledgements: Participation in this study leaves the physicians with many acknowledgements for excellent and expert assistance. From Fisons plc, Pharmaceutical Division, the authors wish to thank A. Benbow, S. Bustacchini, M. Degeyter, V. Elegant, A. Harper, J. Lewis, H. Medley, A. Wardle, and M. Wegar for their enthusiasm and contribution to the design, conduct and analysis of this study. We also wish to acknowledge the contribution of A. van As (USA). This study was supported by Fisons plc, Pharmaceutical Division, who also supplied the nedocromil sodium and placebo treatments. Part of this study has appeared as a Short Communication in Family Practice (1991; 8: 402-403) following presentation at the Functional Status Assessment International Family Medicine Research Workshop, Nijmegen, The Netherlands (April 1991).

\section{References}

1. Jones PW. - Quality of life measurement for patients with diseases of the airways. Thorax 1991; 46: 676-682.

2. Bergner M, Bobbitt RA, Carter WB, Gilson BS. - The Sickness Impact Profile: development and final revision of a health status measure. Med Care 1981; 19: 878-885.

3. Kaplan RM, Atkins CJ, Timms R. - Validity of a quality of well-being scale as an outcome measure in chronic obstructive pulmonary disease. J Chron Dis 1984; 37 : 85-95.

4. Jones PW, Baveystock CM, Littlejohns P. - Relationships between general health measured with the Sickness Impact Profile and respiratory symptoms, physiological measures and mood in patients with chronic airflow limitation. $\mathrm{Am}$ Rev Respir Dis 1989; 140: 1538-1543.

5. MacKenzie CR, Charlson ME, DiGioa DD, Kelley K. Can the Sickness Impact Profile measure change? An example of scale assessment. J Chron Dis 1986; 39: 429-438.

6. Tandon PK, Stander H, Schwartz RP. - Analysis of quality of life data from a randomized placebo-controlled heart failure trial. J Clin Epidemiol 1989; 42: 955-962.

7. Jones PW, Quirk FH, Baveystock CM. - The St George's Respiratory Questionnaire. Respir Med 1991; 85 (Suppl. B): 25-31.

8. Jones PW, Quirk FH, Baveystock CM, Littlejohns P. A self-complete measure for chronic airflow limitation - the St George's Respiratory Questionnaire. Am Rev Respir Dis 1992; 145: 1321-1327.

9. Zigmond AS, Snaith RP. - The Hospital Anxiety and Depression scale. Acta Psychiatr Scand 1983; 67: 361-370.

10. Carrasco E, Sepulveda R. - The acceptability, safety and efficacy of nedocromil sodium in long-tern clinical use in patients with perennial asthma. J Int Med Res 1988; 16: 394-401.

11. Cherniack RM, Wasserman SI, Ramsdell JW, et al. -
A double-blind multicenter group comparative study of the efficacy and safety of nedocromil sodium in the management of asthma. Chest 1990; 96: 1299-1306.

12. Bone MF, Kubik MM, Keaney NP, et al. - Nedocromil sodium in adults with asthma dependent on inhaled corticosteroids: a double-blind, placebo-controlled study. Thorax 1989; 44: 654-659.

13. Callaghan B, Teo NC, Clancy L. - Effects of the addition of nedocromil sodium to maintenance bronchodilator therapy in the management of chronic asthma. Chest 1992; 101: 787-792.

14. Bergner M. - The Sickness Impact Profile. In: Walker SR, Rosser RM, eds. Quality of Life: Assessment and Application. Boston, M.T.P., 1987; pp. 79-94.

15. Quirk FH, Jones PW. - Patients' perception of distress due to symptoms and effects of asthma on daily living and an investigation of possible influential factors. Clin Sci 1990; 79: 17-21.

16. Quirk FH, Baveystock CM, Wilson RC, Jones PW. Influence of demographic and disease related factors on the degree of distress associated with symptoms and restrictions on daily living due to asthma in six countries. Eur Respir J 1991; 4: 167-171.

17. Snedecor GW, Cochran WG. - Statistical Methods. (6th edn). Ames, Iowa State University Press, 1967.

18. Intermittent positive pressure trial group. - Intermittent positive pressure breathing therapy of chronic obstructive pulmonary disease. Ann Intern Med 1983; 99: 612-620.

19. Nocturnal oxygen therapy trial (NOTT) group. - Continuous or nocturnal oxygen therapy in hypoxemic chronic obstructive lung disease. Ann Intern Med 1980; 93: 391-398.

20. Juniper EF, Guyatt GH, Epstein RS, Ferrie PJ, Jaeschke R, Hiller TK. - Evaluation of impairment of healthrelated quality of life in asthma: development of a questionnaire for use in clinical trials. Thorax 1992; 47: 76-83.

21. Larson JL, Kim MJ, Sharp JT, Larson DA. - Inspiratory muscle training with a pressure threshold breathing device in patients with chronic obstructive pulmonary disease. Am Rev Respir Dis 1988; 138: 689-696.

22. Hoffman LA, Wesmiller SW, Sciurba FC, et al. - Nasal cannula and transtracheal oxygen delivery. A comparison of patient response after 6 months of each technique. Am Rev Respir Dis 1992; 145: 827-831.

23. Yellowlees PM, Kalucy RS. - Psychobiological aspects of asthma and the consequent research implications. Chest 1990; 97: 628-634.

24. Wells A, Drennan C, Holst P, Jones D, Rea H, Thornley P. - Comparison of nedocromil sodium at two dosage frequencies with placebo in the management of chronic asthma. Respir Med 1992; 86: 311-316.

25. Fairfax AJ, Allbeson M. - A double-blind group compara-tive trial of nedocromil sodium and placebo in the management of bronchial asthma. J Int Med Res 1988; 16: 216-224.

26. Fyans PG, Chatterjee PC, Chatterjee SS. - Effects of adding nedocromil sodium (Tilade ${ }^{\circledR}$ ) to the routine therapy of patients with bronchial asthma. Clin Exp Allergy 1989; 19: 521-528.

27. Stevens MT. - Approach to designing a clinical study and assessing a clinical paper. Asthma symposium. Montreal, Quebec, Rubicon Publishing Inc. Ontario, 1990; 52-64.

28. Burney PGJ, Laitinen LA, Perdrizet S, et al. - Validity and repeatability of the IUATLD (1984) Bronchial Symptoms Questionnaire: an international comparison. Eur Respir J 1989; 2: 940-945. 